\title{
Application of Unmanned Aerial Vehicle Remote Sensing in Engi- neering Measurement
}

Qing Li*

Hangzhou Pengcheng Remote Sensing Technology Co., Ltd. E-mail: liqing@sina.com

\begin{abstract}
Unmanned aerial vehicle remote sensing is widely used in the whole engineering measurement in recent years. It has many advantages including simple operation, high accuracy and high efficiency. It is precisely because of these advantages that unmanned aerial vehicle remote sensing has gradually replaced the traditional surveying and mapping technology to be widely used. With the continuous expansion of the number and scale of projects in China, the effect of unmanned aerial vehicle remote sensing in engineering measurement is getting bigger and bigger. This article mainly analyzes the advantages of unmanned aerial vehicle remote sensing and its application in engineering measurement, so as to provide some reference for the development of surveying and mapping engineering in China.
\end{abstract}

Keywords: Unmanned Aerial Vehicle Remote Sensing; Surveying and Mapping Engineering; Specific Application Analysis

\section{Unmanned aerial vehicle remote sensing in the specific application of engineering measurement}

\subsection{Application in urban planning survey}

At present, China's urbanization process is developing very rapidly, and the overall urbanization population is also growing rapidly. The biggest problem in recent years is that the urban area can't meet people's needs. So in this case, it is necessary to improve the scientificity and rationality of the whole urban planning and realize the good development of the city. In the whole urban planning survey by unmanned aerial vehicle remote sensing, good results can be achieved. Through engineering surveying and mapping, reliable basic data can be obtained. By building a digital ground model, the staff can realize the visual presentation of the whole urban planning. This mapping technology can also obtain complete geographic information, provide clear image data for urban planning, and also provide reliable data reference for the planning and construction of relevant personnel. However, when the unmanned aerial vehicle remote sensing is used in urban planning, the staff must always pay attention to the flight situation and image data of unmanned aerial vehicles. At the same time, they should constantly eliminate and screen the relevant information and data, and fully consider the flight speed and angle of unmanned aerial vehicles, so as to better improve the accuracy and precision of engineering surveying and mapping, meet the development needs of engineering planning and construction in China, and realize the long-term development of cities.

\subsection{Application in obtaining image data}

In the specific development process of surveying and mapping engineering, designers and constructors

Copyright (C) 2020 Qing Li

doi: 10.18282/rs.v9i2.1372

This is an open-access article distributed under the terms of the Creative Commons Attribution Non-Commercial License

(http://creativecommons.org/licenses/by-nc/4.0/), which permits unrestricted non-commercial use, distribution, and reproduction in any medium, provided the original work is properly cited. 
must make full use of unmanned aerial vehicle remote sensing according to the actual situation, and apply it scientifically to practical work. In actual work, in order to improve the application effect of this technology and ensure the accuracy and validity of the results, the actual topography and geomorphology of the measurement area should be taken as the basic content, and on this basis, the staff should carry out scientific design and arrangement for the flight platform and route combining with the actual situation. After the design of the whole project is completed, it is necessary to carry out the test flight acceptance, which can not only ensure the rationality and scientificity of the selection and utilization of the equipment platform, but also realize the acquisition and collection of surveying and mapping image data, and can utilize various surveying and mapping image data. If it is required to ensure the timeliness and effectiveness of image data acquisition, the staff must also adopt scientific and reasonable unmanned aerial vehicle remote sensing according to the actual situation. Practice has proved that there is a big difference between unmanned aerial vehicle remote sensing and the traditional remote sensing technology in the acquisition of image data. The fundamental reason for this difference is that there will be certain deviation when unmanned aerial vehicle remote sensing is used, both in amplitude and corner. Therefore, in the specific application process of this technology, it can fundamentally solve the problem that it is difficult to obtain image data. In addition, in the specific development process of surveying and mapping engineering, it is necessary to make full and reasonable use of unmanned aerial vehicle remote sensing, adjust flight attitude in time, and make compensation by making up shots, etc., so as to ensure that the original images are captured by digital cameras to the maximum extent, to ensure the basic standards of image processing, and to ensure the authenticity and validity of surveying and mapping engineering data.

\subsection{Application in data collection}

According to different subjects of data acquisition, unmanned aerial vehicle data acquisition can be divided into manual acquisition and automatic acquisition. Manual data acquisition mainly uses computer remote control technology, and the unmanned aerial vehicle operators are inside the base station, and take selective images of the unmanned aerial vehicle according to the actual data acquisition needs, so as to obtain all kinds of data information that people need. Automatic encryption is a self-protection mechanism of unmanned aerial vehicle internal control system. After receiving the image information needed, unmanned aerial vehicle shooting equipment and sensors can temporarily store this part of information. In order to better ensure the security of data storage, certain encryption programs need to be set for the memory. When managers need to call the unmanned aerial vehicle to shoot images, they need to access them according to the access rights, so as to better ensure the privacy of the data and ensure the accuracy of the data to a certain extent.

\subsection{Application in data processing}

Compared with the traditional manual data processing mode, the processing efficiency and quality of unmanned aerial vehicle in data processing have been greatly improved. Under normal circumstances, unmanned aerial vehicle remote sensing can't form a separate product, which must be processed by corresponding software. Unmanned aerial vehicle remote sensing's image level is not high, and the number of images is not large. The inclination angle and regularity of each image are not perfect, and automatic connection and assembly can not be realized directly. In this case, it is necessary to acquire image data by using unmanned aerial vehicle technology. First of all, download the image data obtained by unmanned aerial vehicle to the data processing system, and organize and match these data continuously. At the same time, combine GPS technology to obtain more accurate image internal and external orientation elements. Workers must also carry out radiation processing and color correction on the images to obtain high-quality results.

\subsection{Application in mine survey}

In order to ensure the quality and safety of mine development, the staff must carry out the overall surveying and mapping management of the mine before it is carried out. However, because of the particularity of the mine, it is not feasible to use the traditional method to carry out engineering measurement. Traditionally, the staff carry working equipment to inspect it, but some equipment cannot be safely transported to the mining 
area. Moreover, the ecological environment of the whole mining area is very bad and the road is not smooth enough, which will easily cause serious impact on the personal safety of the staff. In order to fundamentally avoid these problems, it is necessary to ensure the quality of geological surveying and mapping work. Workers can use unmanned aerial vehicle surveying and mapping technology to survey mines, which can give full play to the advantages of unmanned aerial vehicle remote sensing, reduce the workload of workers and improve their work quality and efficiency. In the process of mine survey, in addition to the unmanned aerial vehicle remote sensing, ultra-high definition imaging equipment can be used to quickly map and shoot the mining area. For some mine areas that workers can't enter, they can also control drones to map the whole area. After analyzing various information coordinates through computer measurement system and calculation management, the accuracy and validity of mapping data can be greatly guaranteed. China's unmanned aerial vehicle remote sensing has made great contributions in the development process, and its effect is great. The effect of low-altitude remote sensing system is very powerful, while the cost is relatively low. The operation is simple and flexible, which effectively overcomes various problems.

\section{Analysis of the problems in the measurement process of unmanned aerial vehicle}

Although the measurement process of unmanned aerial vehicle has many advantages, such as flexible operation and convenient control, it puts forward higher requirements for the endurance of the unmanned aerial vehicle itself because of the long flight operation time, especially when performing a wide range of remote sensing operations. Therefore, people have higher requirements for various functions of the unmanned aerial vehicle. For example, in the process of information collection, the original image shooting is gradually changed to the current video recording conversion. In order to better meet the development requirements of the new era, it is necessary to carry more functional equipment on the unmanned aerial vehicle and increase the pollution-free flight load. At present, how to quickly improve the endurance of unmanned aerial vehicle is a major problem in unmanned aerial vehicle remote sensing measurement in China.

\subsection{The take-off and landing and wind re- sistance of drones to be improved}

In the process of surveying and mapping some mountain forests with poor natural conditions, it is necessary for unmanned aerial vehicles to have good take-off and landing performance. For many large-scale unmanned aerial vehicles that are downhill landing, if they can't finish good take-off and landing in the real environment, the functional effect of unmanned aerial vehicles will be damaged to a certain extent. There will even be no place that can support the take-off conditions of large-scale unmanned aerial vehicles. In the measurement, if small unmanned aerial vehicles are selected, all kinds of unfavorable factors can be avoided. However, the flying height of small unmanned aerial vehicles is relatively low, which is greatly affected by wind, and it is easy to have a great impact on the accuracy of the measurement results. When designing unmanned aerial vehicles, if designers want to increase the wind resistance, they usually solve the problem by increasing the weight of unmanned aerial vehicles. However, if the weight of unmanned aerial vehicles is pursued too much in design, the take-off and landing on the site will not meet the specified standard requirements. Moreover, during the measurement work, various remote sensing images obtained by small unmanned aerial vehicles do not have aerial coordinates, which greatly reduces the accuracy of the images. In some cases, they even have to take artificial points to carry out engineering measurement.

\subsection{Easily affected by high-altitude airflow}

Some unmanned aerial vehicles can't fly smoothly due to the influence of high-altitude airflow, which makes the unmanned aerial vehicle deviate from the original route, makes the monitoring results not meet the specified requirements, and the shooting results not accurate enough. These problems will hinder the development and application of unmanned aerial vehicle remote sensing in China. It is believed that in the future, through the continuous research and development of scientific researchers, unmanned aerial vehicle remote sensing can actively overcome these shortcomings and problems and better promote its long-term development in China.

\subsection{Flight attitude to be improved}


Due to some technical requirements, the traditional unmanned aerial vehicle can't carry sensors with high accuracy, which will have a great impact on the development of measurement work. Using this equipment, it can't obtain images with high accuracy, or it can't meet the drawing requirements of large scale terrain. Therefore, for new surveying and mapping, it is necessary to invent a sensor suitable for unmanned aerial vehicle according to the actual situation. When the unmanned aerial vehicle is working, it must be controlled so that it can carry out engineering measurement according to the originally set process and better meet the measurement technical requirements.

\section{Conclusion}

Compared with the traditional measurement technology, by adopting unmanned aerial vehicle remote sensing, the accuracy of the whole engineering measurement data results can be greatly improved, and the construction quality of the project can be improved. In addition, for some natural environments with harsh geological conditions, the engineering measurement is greatly facilitated, with the risk of workers reduced, and the work quality improved. It is precisely because unmanned aerial vehicle itself has many advantages that it has been widely used in engineering measurement. Relevant staff must also apply this efficient monitoring technology to more surveying and mapping work according to the actual situation, so as to better improve the effect and quality of engineering measurement in China.

\section{References}

1. Cao W. Application of unmanned aerial vehicle remote sensing in engineering measurement (in Chinese). Quality and Market 2020; (6): 61-64.

2. Zheng S. Application of unmanned aerial vehicle remote sensing in surveying and mapping engineering measurement (in Chinese). Smart City 2020; 6(5): 56-57. 